\title{
Understanding the mechanisms of entrapment neuropathies
}

\author{
Review article \\ Khoa Pham, B.S., ${ }^{1}$ and Ranjan Gupta, M.D. ${ }^{1-3}$ \\ Departments of ${ }^{1}$ Orthopaedic Surgery, ${ }^{2}$ Anatomy \& Neurobiology, and ${ }^{3}$ Biomedical Engineering, \\ University of California, Irvine, California
}

\begin{abstract}
Compression neuropathies are highly prevalent, debilitating conditions with variable functional recovery following surgical decompression. Due to the limited amount of human nerve tissue available for analysis, a number of animal models have been created to help investigators understand the molecular and cellular pathogenesis of chronic nerve compression (CNC) injury. Evidence suggests that CNC injury induces concurrent Schwann cell proliferation and apoptosis in the early stages of the disorder. These proliferating Schwann cells downregulate myelin proteins, leading to local demyelination and remyelination in the region of injury. In addition, the downregulation of myelin proteins, in particular myelin-associated glycoprotein, allows for axonal sprouting. Interestingly, these changes occur in the absence of both morphological and electrophysiological evidence of axonal damage. This is in direct contrast to acute injuries, such as transection or crush, which are characterized by axonal injury followed by Wallerian degeneration. Because the accepted trigger for Schwann cell dedifferentiation is axonal injury, an alternate mechanism for Schwann response must exist in CNC injury. In vitro studies of pure Schwann cells have shown that these cells can respond directly to mechanical stimuli by downregulating myelin proteins and proliferating. These studies suggest that although the reciprocal relationship between neurons and glial cells is maintained, chronic nerve compression injury is a Schwann cell-mediated disease. (DOI: 10.3171/FOC.2009.26.2.E7)
\end{abstract}

\section{KEY WoRdS - carpal tunnel syndrome - chronic nerve compression - nerve entrapment $\quad$ Schwann cell}

$\mathrm{C}$ HRONIC nerve compression injuries affect millions of individuals. Yet despite their prevalence, there has been little progression in the understanding of compression injuries until recently. Patients with carpal tunnel syndrome, the most common form of CNC injury, present with sensory abnormalities within the distribution of the median nerve, including numbness, tingling, and pain. If left untreated, these symptoms often result in permanent sensory and motor loss. Conservative treatments for carpal tunnel syndrome include splinting the wrist in a neutral position, ultrasound therapy, and corticosteroid treatment. Surgical decompression of the nerve is warranted if symptoms are refractory to conservative treatments. ${ }^{8}$ However, the outcomes of decompression may be unpredictable, and the reversal of motor weakness is usually limited. Therefore, an increase in the understanding of the pathological mechanisms is warranted.

\footnotetext{
Abbreviations used in this paper: CMAP = compound motor action potential; $\mathrm{CNC}=$ chronic nerve compression; $\mathrm{DRG}=$ dorsal root ganglion; GAP-43 = growth-associated protein-43; GDNF = glial cell line-derived neurotrophic factor; $\mathrm{MAG}=$ myelin-associated glycoprotein.
}

The structure of normal, uninjured myelinated nerve fibers has been a topic of intense research. It is known that in myelinated peripheral nerves, a single Schwann cell will associate with a single segment of axon and extend cytoplasmic processes around it. This will create a segment of myelinated axon known as the internode. At the edges of the internode, the myelin begins to form distinctive folds that are characteristic of juxtaparanodal and paranodal regions. Interrupting the internodal segments at regular intervals and immediately adjacent to paranodes are the nodes of Ranvier, which are areas of axon devoid of myelin (Fig. 1). ${ }^{32}$ Functionally, the juxtaparanode and node of Ranvier are physiologically important, because the juxtaparanode contains high concentrations of voltage-gated potassium channels, and the node of Ranvier contains high concentrations of voltage-gated sodium channels. Following an excitatory stimulus of sufficient magnitude, sodium ions enter the axon, followed by the exit of potassium ions as the action potential propagates down the length of the axon. The subcellular localization of potassium and sodium channels to the juxtaparanode and node of Ranvier, respectively, and the insulating properties of myelin limit the exchange of ions between 


\section{K. Pham and R. Gupta}
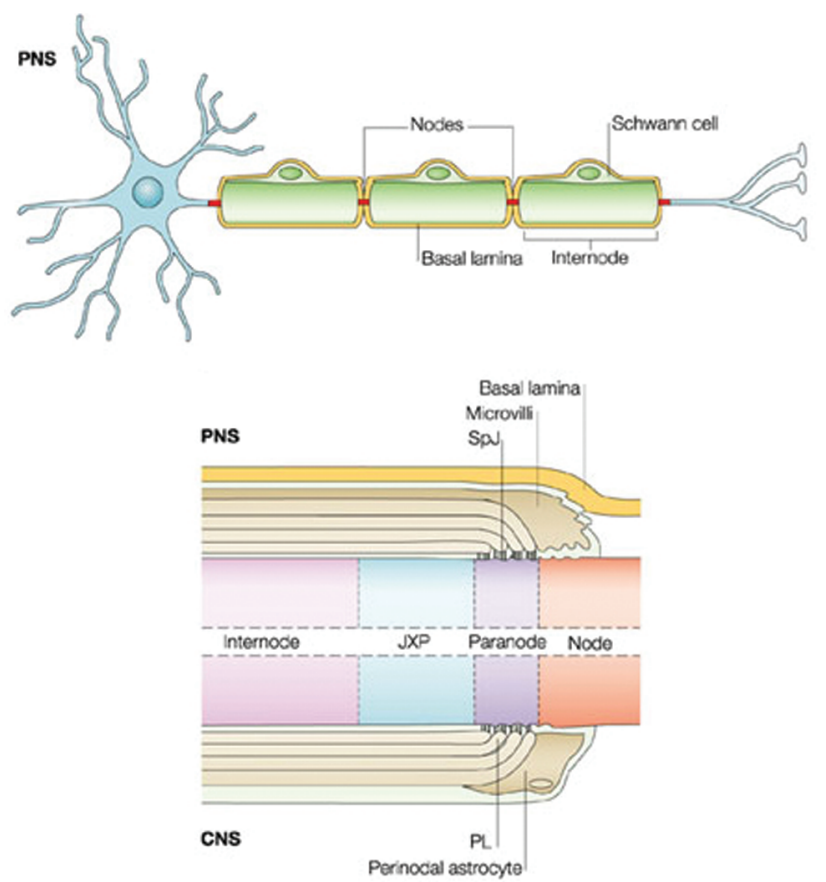

Nature Reviews | Neuroscience

FIG. 1. Schematic drawing of the internode, juxtaparanode (JXP), paranode, and node of Ranvier in relation to the myelinated axon. Voltage-gated sodium channels are found at the node of Ranvier, whereas voltage-gated potassium channels are found at the juxtaparanode. CNS = central nervous system; PL = paranodal loop; PNS = peripheral nervous system; SpJ = septatelike junction. Reprinted with permission from Qiu, J: Cell biology of the neuron: scaffolding at the nodes. Nature Reviews Neuroscience 5:823, 2004.

the axon and the extracellular environment to these areas. As a result, this allows the action potential to travel faster down the axon via saltatory conduction. ${ }^{32}$ Following injury, there is reorganization of this basic structure, with one of the most prominent changes being an increase in the average g-ratio (axon diameter/total fiber diameter) from $\sim 0.6$ to 0.8 in rodent models of $\mathrm{CNC}$.

Although compression neuropathies are fairly ubiquitous, the characterization of the changes that occur in the compressed nerve has been difficult because it is unethical to harvest human nerve tissue due to the ensuing permanent donor-site morbidity that would occur. In a postmortem study of subclinical entrapment of the median and ulnar nerves, a thickening of the endoneurium, perineurium, and epineurium was noted. Fibers teased from these nerves revealed thinning and retraction of the myelin and intercalated segments indicative of previous demyelination..$^{26}$ Limited human studies on compressed sensory nerves that were removed to treat refractory pain have revealed a similar thinning of the myelin and thickening of the endoneurium, microvessels within the endoneurium, and perineurium in the region of compression. ${ }^{21}$ Further characterization of the pathological mechanisms following $\mathrm{CNC}$ injury, however, has proved to be difficult when using human tissue.

The limited amount of human nerve tissue available for analysis has spurred the creation of a number of ani-

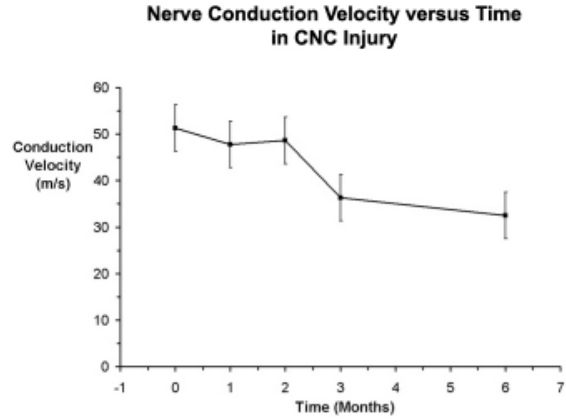

FIG. 2. Graph showing nerve conduction velocity gradually decreasing following CNC injury. Note that there is no change in nerve conduction velocity in the early stages postcompression. At each time point, the mean values are shown with their standard deviations.

mal models to understand the pathological features of CNC. One such animal model uses a biologically inert Silastic tube placed around the sciatic nerve of SpragueDawley rats..$^{14,28}$ The tube is of sufficient diameter to allow for gliding along the nerve and to avoid any type of acute mechanical deformation of the nerve. Alternatively, a rabbit model for $\mathrm{CNC}$ has been created in which an angioplasty catheter is inserted into the carpal tunnel and used to increase the intracarpal pressure by amounts that can be specified by the investigator. ${ }^{6}$ Additional methods have involved compressing the nerve through inflammation of the surrounding tissue. One such method involves chemically inducing inflammation within the carpal tunnel to form exuberant granulation tissue. ${ }^{34}$ Other investigators have induced compression of the median nerve through repeated electrical stimulation of the flexor digitorum profundus muscle or coaxing the animal into repeated wrist movements. ${ }^{39}$ Because the subsinovial connective tissue of the wrist is believed to be important in the pathogenesis of CNC by some investigators, the mechanical properties of the subsinovial connective tissue have been characterized in both human cadavers and rabbit models. ${ }^{45,46}$ Although they are all very different, each animal model shares certain characteristics that are consistent with the human condition; principal among these is a progressive, gradual decline in nerve conduction velocity (Fig. 2).

Whereas chronic nerve injuries are undercharacterized, there has been a large amount of work done on acute neural injuries such as a transection or crush injury. Following transection, the axonal cytoskeleton disintegrates and the axonal membrane breaks apart. ${ }^{5}$ Axonal degeneration is followed by rapid recruitment of hematogenously derived macrophages, leading to degradation of the myelin sheath. ${ }^{9}$ This cascade of events following axonal injury has been termed Wallerian degeneration. Secondary to Wallerian degeneration, Schwann cells undergo a programmed dedifferentiation, including the downregulation of myelin proteins and cellular proliferation that ultimately leads to demyelination..$^{42}$ These reprogrammed Schwann cells form "bands of Büngner," which provide a cellular substrate for nerve regeneration and subsequent remyelination. ${ }^{36}$ Recent work has identified c-jun as a major transcription factor for Schwann cell dedifferentiation. ${ }^{29}$ In normal nerves, promyelination transcription factors such as krox-20 (Egr2) initiate and maintain 


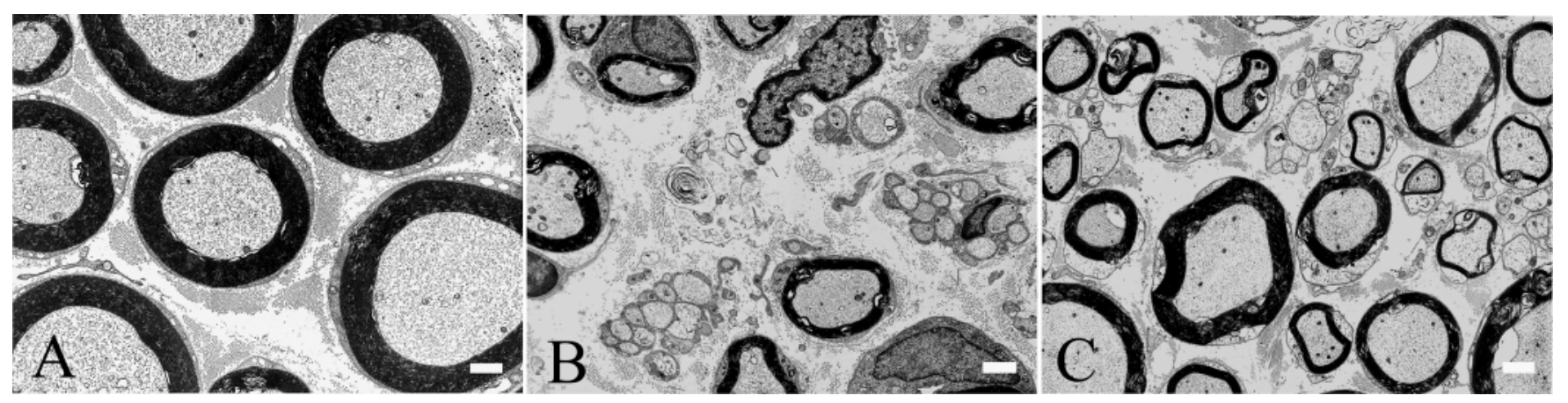

FIG. 3. Electron micrographic images of sections of rat sciatic nerves obtained in control animals (A), after 1 month of compression (B), and after 8 months of compression (C). The 1-month compressed nerves show decreased myelin thickness with intact healthy axons, with a significant increase in the number of Schwann cells and unmyelinated axons. Pathological features were localized to the periphery of the nerve; the center remained normal. The 8-month compressed nerves show a similar decrease in myelin thickness; however, the increased number of unmyelinated axons is not present. Bar $=1 \mu \mathrm{m}$.

myelination. ${ }^{18,43}$ Following injury, the increase in c-jun is accompanied by a concomitant decrease in krox- 20 . The antagonistic relationship between krox-20 and c-jun modulate the transcription factor sox-2, which maintains many cell types, including Schwann cells, in an undifferentiated state. ${ }^{18}$ Due to the gradual nature of CNC injury, it has previously been characterized as a mild form of Wallerian degeneration. As recent experimental data have shown, the pathogenesis of acute transection and that of $\mathrm{CNC}$ injuries are quite distinct. Nevertheless, the wealth of knowledge regarding transection injuries has been an invaluable framework for understanding the cellular and molecular pathogenesis of compression neuropathies.

\section{Demyelination and Remyelination}

It has been hypothesized that the change in nerve conduction velocity seen in CNC is due to gradual demyelination followed by subsequent remyelination of the compressed nerve. As analysis of compressed human nerve segments has revealed marked thinning of myelin and axonal profiles consistent with demyelinating and remyelinating fibers, ${ }^{21}$ animal models for $\mathrm{CNC}$ have described similar thinning of the myelin beginning in the periphery of the nerve (Fig. 3). ${ }^{12,28}$ The appearance of new, thinner myelin is considered to be a marker of remyelination by Schwann cells, and thus it is clear that CNC induces demyelination followed a wave of remyelination. ${ }^{19}$

Individual fibers teased from the periphery of the nerve have revealed a unique pattern of demyelination along the length of the axon. ${ }^{12}$ The myelin changes initially occur at the paranode, where the myelin is thought to be most unstable, and progress toward the internode. Consistent with myelin degeneration, ovoidlike structures were noted in normal paranodal regions adjacent to areas of demyelination. In addition, regions of thin myelin were found to be interspersed between regions of normal myelin. These regions were also found to have internodes of shorter lengths and to be associated with proliferating Schwann cells. Furthermore, teased nerve fibers were found to have an increase in the number of SchmidtLanterman incisures. ${ }^{1}$ Although their function remains a subject of much debate, these incisures are likely to assist in the metabolic processes of the myelin sheath, including growth and maintenance. ${ }^{44}$ The increase in SchmidtLanterman incisures along the axon thus most likely represents an increase in metabolic demand secondary to demyelination and remyelination in CNC injury. These findings in both human nerve tissue and animal models support the hypothesis that the decrease in nerve conduction velocity is a result of a new population of remyelinated fibers with thinner myelin and decreased internodal lengths.

\section{Concurrent Proliferation and Apoptosis of Schwann Cells}

In an established animal model for CNC injury, Schwann cells have been found to proliferate in both the compressed and distal nerve segments at 2 weeks after CNC injury (Fig. 4). The peak of proliferation is reached at 1 month, representing a 6-fold increase in Schwann cell number. Remarkably, during this period of proliferation there is a concomitant increase in the level of Schwann cell apoptosis (Fig. 5). ${ }^{14}$ The observed apoptosis is biphasic, with one phase occurring at the same time as proliferation and the other occurring in the late stages. Late-stage apoptosis is presumably due to the connective tissue hypertrophy and a gradual loss of axons, but the cause of early-stage apoptosis (and also proliferation) is unknown. As with demyelination, proliferation and apoptosis of Schwann cells is initially found in the periphery of the nerve. Interestingly, these processes occur at a time when there is no evidence of axonal damage or Wallerian degeneration, both of which have been shown to induce Schwann cells to proliferate.

\section{Downregulation of Myelin-Associated Proteins and Axonal Sprouting}

In adults, Schwann cells have a function in the maintenance of myelin, in part, through the expression of myelin proteins. Following CNC injury, Schwann cells in the compressed region downregulate MAG as part of the program of demyelination. ${ }^{13}$ Analysis of electron micrographs at the same time point reveal an increase in the 

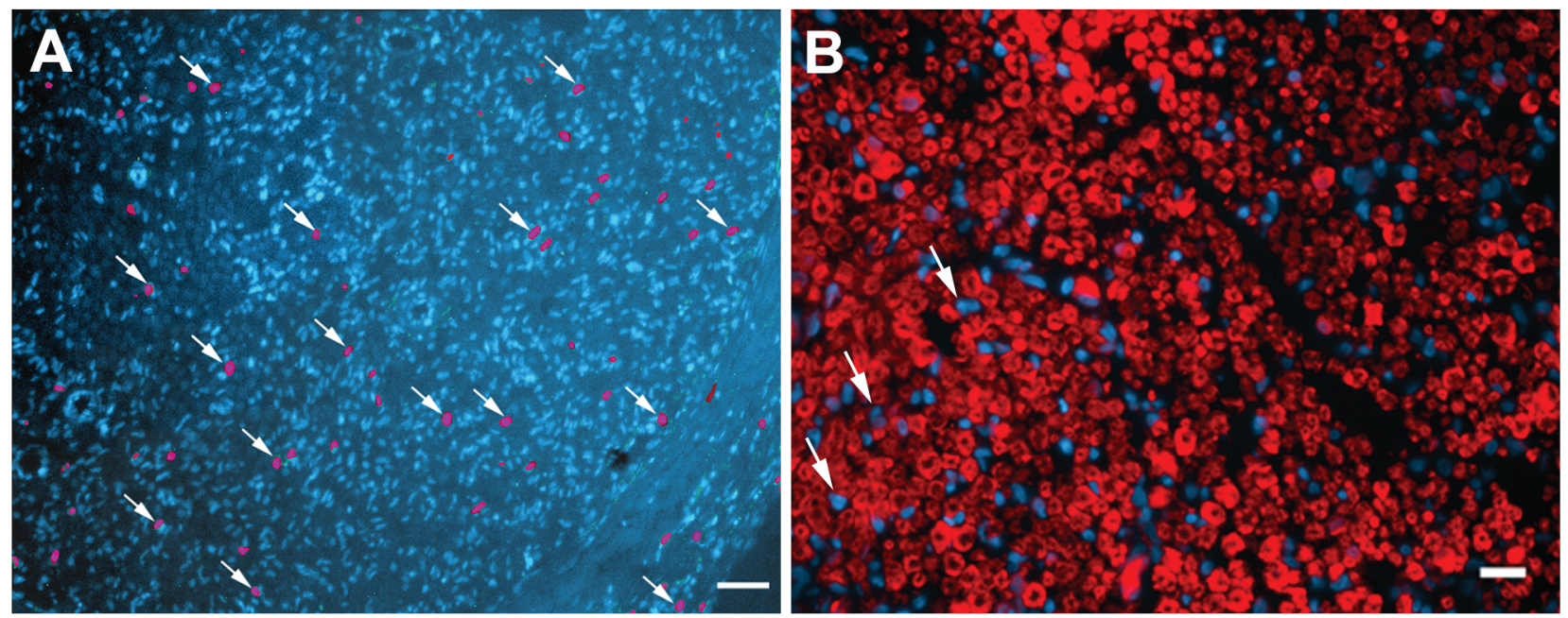

FIG. 4. Photomicrographs showing cells after CNC. A: Bromodeoxyuridine labeling of proliferating cells (red) in the compressed nerve at 2 weeks postcompression. Arrows indicate selected positive Schwann cell nuclei. B: The S100 staining (red) of a nerve cross-section after 1 month of compression shows that the proliferating cells (arrows) are indeed Schwann cells. Bar $=50 \mu \mathrm{m}(\mathrm{A})$ and $20 \mu \mathrm{m}(\mathrm{B})$

number of small unmyelinated fibers grouped together by nonmyelinating Schwann cells into structures known as Remak bundles, which is consistent with axonal sprouting (Fig. 3).$^{13}$ In children, MAG has been demonstrated to enhance axonal growth. ${ }^{17}$ In adults, however, MAG is to known to inhibit axonal regeneration and neurite outgrowth via p75-mediated activation of Rho and binding to the Nogo receptor. ${ }^{7,22,25}$ Therefore, the downregulation of MAG by Schwann cells seems to permit axonal sprouting following $\mathrm{CNC}$ injury. Interestingly, intraneural injections of MAG at the time of injury prevents $\mathrm{CNC}$-induced sprouting, providing further evidence that downregulation of MAG is permissive of axonal sprouting. ${ }^{13}$ As with demyelination and Schwann cell apoptosis/proliferation, axonal sprouting occurs at time when there is no evidence of axonal injury.

\section{The DRG Response to CNC Injury}

Following CNC injury, GAP-43 is upregulated within DRG neurons. ${ }^{4}$ Because GAP-43 is a growth cone molecule critical in the modulation of F-actin behavior to extracellular cues, its upregulation is consistent with the observed axonal sprouting. ${ }^{16}$ Interestingly, GAP-43 upregulation was found to be localized to the cell bodies of small-caliber isolectin B4-binding and calcitonin gene-related peptide-positive neurons. Because these neurons are known to be nociceptive, this potentially explains the onset of nociceptive abnormalities before that of motor abnormalities. Furthermore, there is a transient shift within the populations of sensory neurons within the DRG. ${ }^{4}$ Specifically, there is an increase in the number of isolectin B4-binding and calcitonin gene-related peptide-positive neurons and a corresponding decrease

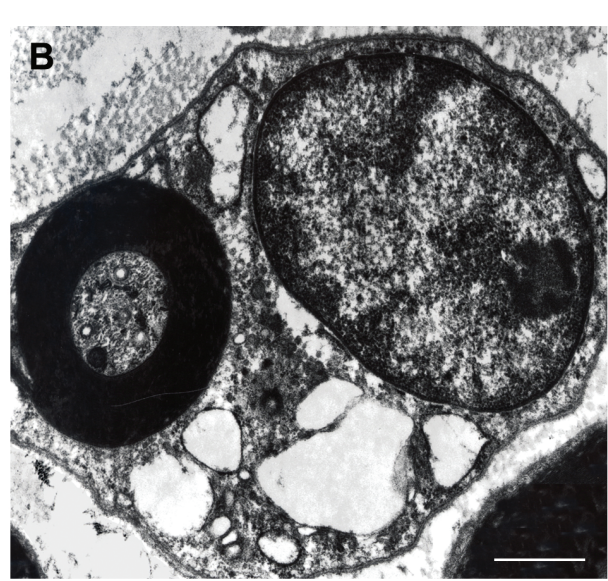

FIG. 5. A: Photomicrograph of the compressed nerve showing marked Schwann cell apoptosis (arrows), as indicated by terminal deoxynucleotidyl transferase::mediated deoxyuridine triphosphate nick-end labeling, at the 2-week time point, primarily within the peripheral aspects of the nerve. B: Electron micrograph of a Schwann cell (identifiable by the surrounding basal lamina and enclosed myelinated nerve) reveals large vacuolar cytoplasmic inclusions and condensed chromatin consistent with apoptosis. Bar $=50 \mu \mathrm{m}(\mathrm{A})$ and $3 \mu \mathrm{m}(\mathrm{B})$. 


\section{Understanding the mechanisms of entrapment neuropathies}
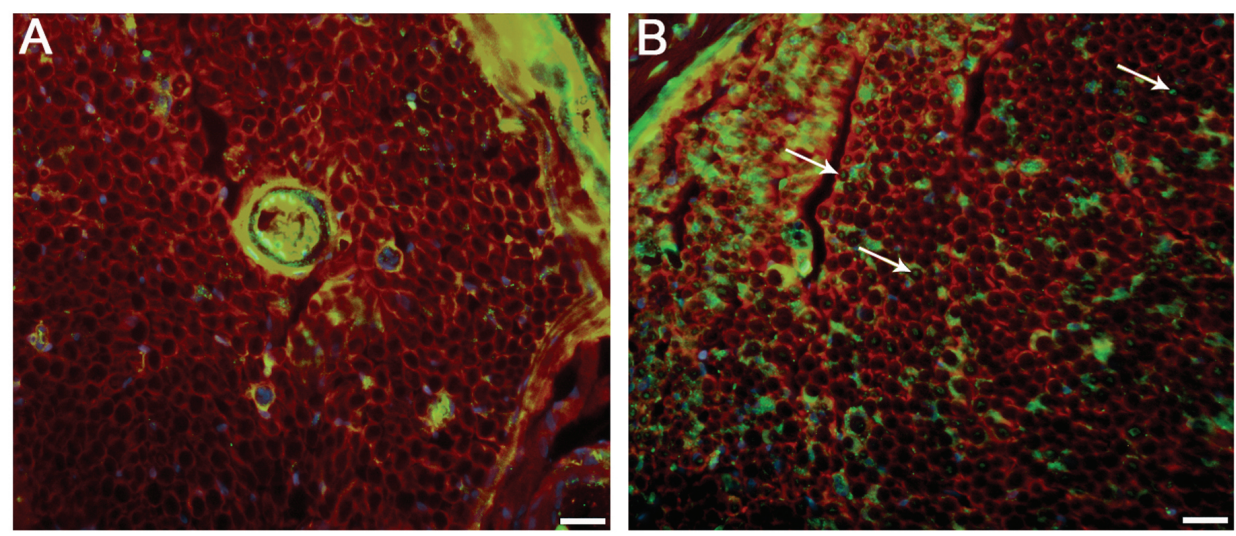

FIG. 6. Immunohistochemical evaluation of normal (A) and compressed (B) sciatic nerve for GDNF protein localization to Schwann cells. The GDNF is represented in green, and S100 protein is in red. Arrows indicate positive GDNF in the center of the axon. Bar $=50 \mu \mathrm{m}$.

in the number of neurofilament-200 neurons. Neurofilament-200 neurons are known to be proprioceptive, and thus a shift from neurofilament-200 neurons to isolectin B4-binding and calcitonin gene-related peptide-positive neurons may represent a mechanism for pain in CNC injury. The cause of the observed phenotypic shift in DRG neurons is most likely a downstream effect of differential growth factor expression by Schwann cells. At the site of the injury, Schwann cells upregulate GDNF and retrogradely transport it to the DRG (Fig. 6). The transient increase in GDNF and phenotypic shift show temporal correlation. Because GDNF is known to be neurotrophic for isolectin B4-binding neurons, ${ }^{23}$ this potentially explains the increase in this neuronal subtype.

\section{Axonal Integrity is Maintained in CNC Injury}

Classically, dedifferentiation of Schwann cells and demyelination in mechanical injuries has been thought to occur secondary to axonal damage. As has been previously mentioned, however, electron microscopic analysis of axons in CNC injury has shown no morphometric evidence of axonal injury. ${ }^{14}$ Rigorous evaluation of axonal integrity following CNC injury has contributed substantial evidence to support this idea. ${ }^{24}$ Motor unit number estimation studies revealed no change in CMAP amplitudes following CNC injury, whereas CMAPs were unattainable in acutely injured nerves (Fig. 7). Analysis of soleus muscle following compression of the sciatic nerve showed no evidence of denervation-induced changes, including areas of grouped muscle fiber atrophy, increased variation in muscle fiber diameter, or the appearance of small angular fibers. Furthermore, axonal injury induces changes within the neuromuscular junction, including degeneration in the presynaptic apparatus, an increase in the density of postsynaptic receptors, and the appearance of extrajunctional receptors. ${ }^{3,31}$ Analysis of neuromuscular junction after CNC injury by using neurofilament and bungarotoxin staining revealed no morphological evidence of neuromuscular junction pathological features consistent with axonal injury (Fig. 8)..$^{24}$ The absence of axonal injury in CNC injury thus suggests the existence of a novel mechanism of Schwann cell dedifferentiation and demyelination.

\section{Macrophage Recruitment Following CNC Injury}

Following CNC injury, there is a gradual infiltration of macrophages over a period of weeks into the compressed and distal segments of the nerve. ${ }^{11}$ This is in sharp contrast to acute injuries, in which a massive wave of macrophage recruitment occurs 24-96 hours after the initial insult. ${ }^{41}$ It has been shown that the primary trigger for macrophage recruitment is an interruption of axonal architecture. Therefore, the primary role of macrophages in acute injuries is to participate in the clearance of axonal and myelin debris. ${ }^{40}$ The absence of axonal injury early after CNC injury may explain the relative lack of
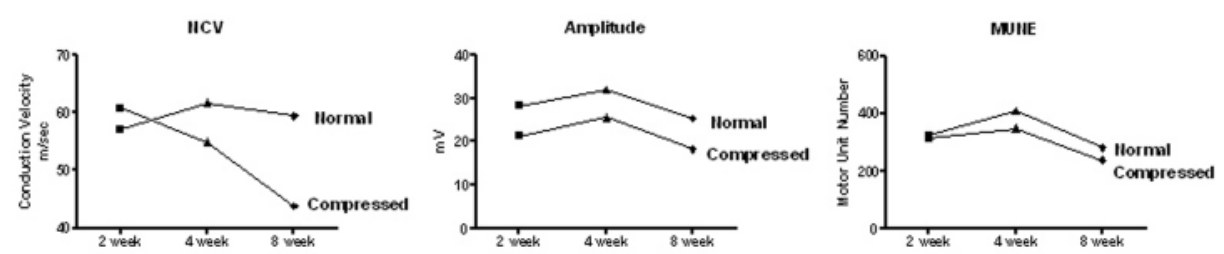

FIG. 7. Graphs showing that nerve conduction velocity (NCV) started declining at 2 weeks and declined by $\sim 20 \mathrm{~m} / \mathrm{s}$ on the compressed side compared with the uncompressed side (curve labeled "normal") in the Silastic tube model for chronic nerve compression in rats. The CMAP amplitude showed a difference between the compressed and uncompressed side at 2 weeks, but never changed further and did not reach statistical significance at any of the time points. Motor unit number estimation (MUNE) also showed no statistically significant difference between normal and compressed sides. 

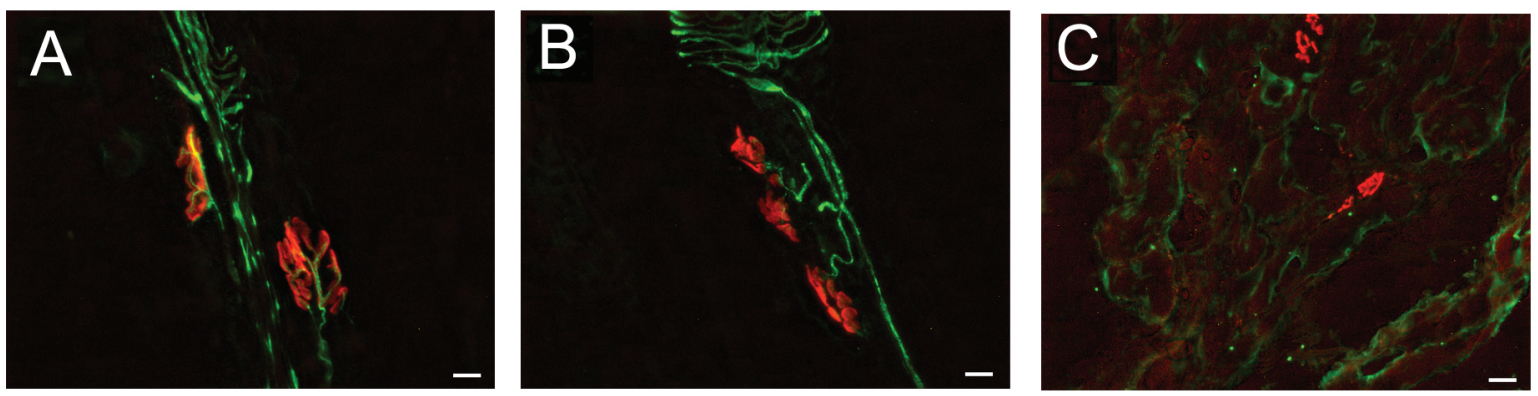

FIG. 8. Photomicrographs showing sections of nerves. Neurofilament and synaptophysin (green) are stained to localize presynaptic elements. Postsynaptic acetylcholine receptors (red) are stained. The neuromuscular junction in rats undergoing CNC of the sciatic nerve remains intact in control (A) and 2-week compressed segments (B). In contrast, animals undergoing transection of the sciatic nerve show extrajunctional acetylcholine receptors and no positive staining for neurofilament or synaptophysin (C). Bar $=100 \mu \mathrm{m}$.

macrophage recruitment. However, because macrophages have also been shown to secrete Schwann cell mitogens (including transforming growth factor- $\beta$; interleukins 1 , $6,10$, and 12 ; and tumor necrosis factor $-\alpha),{ }^{2}$ the possible role of macrophages in Schwann cell proliferation following CNC injury was addressed through macrophage depletion experiments in which clondronate liposomes were used. In the absence of hematogenously derived macrophages, the normal breakdown of the blood-nerve barrier in CNC injury was attenuated (Fig. 9) while the proliferation of Schwann cells was left intact, thus discounting a major role for macrophage-mediated Schwann cell proliferation in the pathological mechanisms of $\mathrm{CNC}$ injury. ${ }^{10}$

\section{Shear Stress Alters the Expression of MAG and Myelin Basic Protein in Schwann Cells}

Because Schwann cell dedifferentiation and demyelination occur independent of axonal damage and macrophage signaling, an alternate mechanism must exist for this process in CNC injury. One possibility is that Schwann cells can respond directly to mechanical stimulus. It has been shown that pure Schwann cell cultures respond robustly to mechanical stimuli in the form of shear stress induced by laminar fluid flow. ${ }^{15}$ Following just 2 hours of shear stress, Schwann cells demonstrated increased proliferation, as evidenced by bromodeoxyuridine uptake and the downregulation of MAG protein and mRNA. The Schwann cell in vitro response to shear stress thus closely mirrors that of the robust in vivo Schwann cell response to compression. Because there are no neurons or other cell types in these cultures, these experiments suggest that Schwann cells can respond to mechanical stimuli independent of axonally derived signals. Nevertheless, the mechanism by which Schwann cells respond to mechanical stimulus is currently unknown. ${ }^{37}$

\section{Conclusions}

Chronic nerve compression injury has been previously characterized as a mild form of Wallerian degenera-
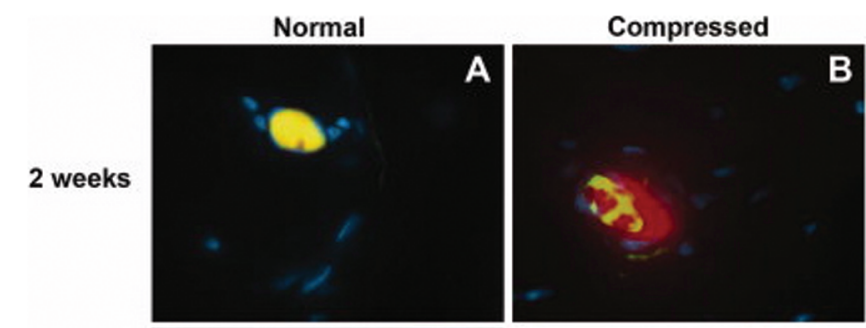

Compressed and Depleted
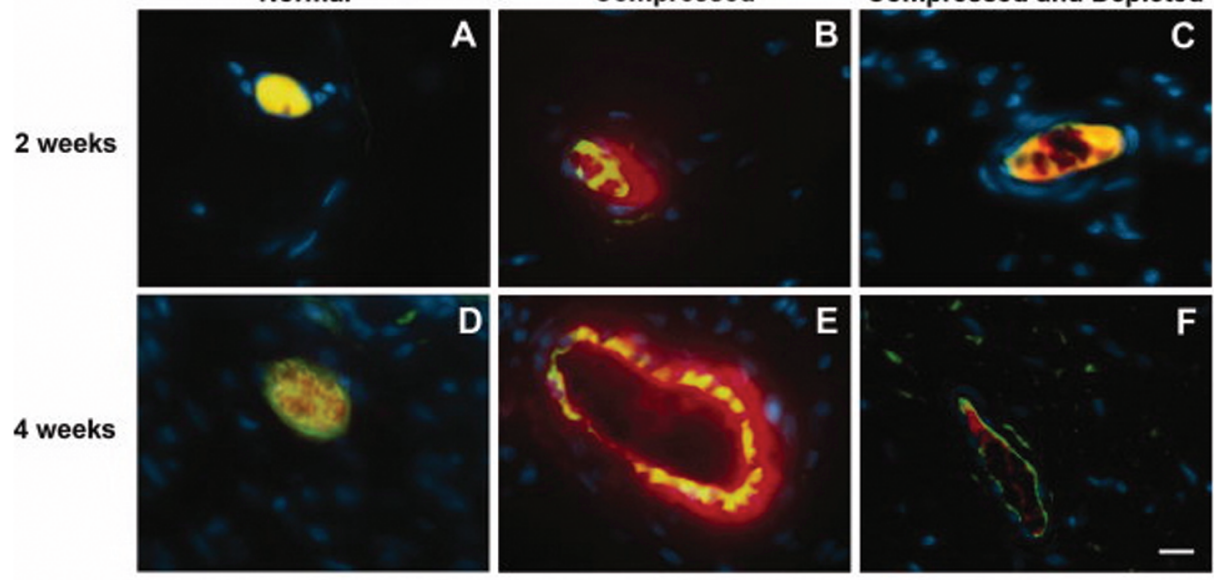

FIG. 9. Photomicrographs showing depletion of hematogenously derived macrophages, which improves the blood-nerve barrier functionality after CNC injury. Immunohistochemical studies show a notable change in blood-nerve barrier permeability following 2 weeks (B) and 4 weeks (E) of CNC injury compared with the contralateral normal nerve (A and D). The depletion of macrophages appears to attenuate this increase in permeability at the same time points ( $\mathrm{C}$ and $\mathrm{F})$. Red represents Evans blue albumin tracer; green represents endothelial cells outlining the lumen of microvessels (RECA-1); and blue represents 4,6'-diamino-2-phenylindole-dihydrochloride nuclear stain. Bar $=10 \mu \mathrm{m}$. 


\section{Understanding the mechanisms of entrapment neuropathies}

tion. In both pathological entities, Schwann cells undergo dedifferentiation resulting in demyelination, downregulation of myelin proteins, and proliferation. However, the precipitating event for Wallerian degeneration is axonal injury, and this is not detected in CNC injury by either morphological or electrophysiological studies. Because there is no axonal injury early after $\mathrm{CNC}$ injury, macrophage recruitment in $\mathrm{CNC}$ injury is not characterized by the massive and immediate increase in macrophage number that is seen in acute injuries. Furthermore, the mitogenic effect of macrophages on Schwann cells does not play a principal role in CNC-induced Schwann cell proliferation. Therefore, the pathogenesis of compression neuropathies is distinct from that of acute injuries and thus cannot be considered a mild form of Wallerian degeneration.

Because the accepted triggers for Schwann cell dedifferentiation are not present, an alternate mechanism must exist. Results of in vitro experiments performed using shear stress suggest that Schwann cells are directly responsive to mechanical stimuli. The mechanisms by which this occurs, however, are currently still unknown. One possibility is that Schwann cell mechanosensitivity is mediated by integrin signaling. Integrins are heterodimeric molecules that function as a bridge between the extracellular matrix and intracellular signaling. Specifically, integrins have been implicated in the mechanotransduction of a variety of mechanical stimuli, including stretch, hydrostatic pressure, shear stress, and osmotic pressure in a variety of different cell types. ${ }^{38}$ It is known that adult myelinating Schwann cells express the $\alpha 6 \beta 4$ integrin, whereas immature and nonmyelinating Schwann cells do not. ${ }^{33} \mathrm{~A}$ recent study of $\beta 4$ knockout mice suggests that the $\alpha 6 \beta 4$ integrin molecule functions in the maintenance of myelin. ${ }^{27}$ It is thus possible that integrin signaling plays a critical role in CNC injury.

\section{Future Directions}

Further study of the mechanisms of demyelination in $\mathrm{CNC}$ injury will require the use of in vitro models. Numerous investigators have shown that applied hydrostatic pressure can influence the activity of many different cell types, including chrondrocytes and osteoblasts. $.^{20,30} \mathrm{Re}-$ cently, a hydrostatic compression chamber model has been described that will allow for the compression of myelinated Schwann cell-DRG cocultures while monitoring critical parameters such as applied pressure, oxygen tension, and $\mathrm{pH}^{35}$ The goal of these chambers is to apply sufficient pressure to elicit a biological response without creating cytotoxicity.

Although recent evidence suggests that Schwann cells are the primary mediators of CNC injury, axonal damage does occur in the later stages of the disease as these patients develop motor weakness. The reciprocal relationship between axons and glial cells is maintained with CNC injury. Yet, in contrast to Wallerian degeneration in which the neuron directs glial cell function, Schwann cells appear to be initially affected by CNC injury and secondarily affect neuronal function. It is likely that a combination of Schwann cell mechanosensitivity and chronic ischemia are part of the pathogenesis of
CNC. An increased understanding of the mechanisms of demyelination and remyelination in $\mathrm{CNC}$ injury through the use of both in vivo and in vitro animal models will allow for the development of novel treatments to improve the outcomes of both nonsurgical and surgical interventions.

\section{References}

1. Berger BL, Gupta R: Demyelination secondary to chronic nerve compression injury alters Schmidt-Lanterman incisures. J Anat 209:111-118, 2006

2. Bruck W: The role of macrophages in Wallerian degeneration. Brain Pathol 7:741-752, 1997

3. Burden SJ, Sargent PB, McMahan UJ: Acetylcholine receptors in regenerating muscle accumulate at original synaptic sites in the absence of the nerve. J Cell Biol 82:412-425, 1979

4. Chao T, Pham K, Steward O, Gupta R: Chronic nerve compression injury induces a phenotypic switch of neurons within the dorsal root ganglia. J Comp Neurol 506:180-193, 2008

5. Chaudhry V, Glass JD, Griffin JW: Wallerian degeneration in peripheral nerve disease. Neurol Clin 10:613-627, 1992

6. Diao E, Shao F, Liebenberg E, Rempel D, Lotz JC: Carpal tunnel pressure alters median nerve function in a dose-dependent manner: a rabbit model for carpal tunnel syndrome. J Orthop Res 23:218-223, 2005

7. Domeniconi M, Cao Z, Spencer T, Sivasankaran R, Wang K, Nikulina E, et al: Myelin-associated glycoprotein interacts with the Nogo66 receptor to inhibit neurite outgrowth. Neuron 35:283-290, 2002

8. Gerritsen AA, de Vet HC, Scholten RJ, Bertelsmann FW, de Krom MC, Bouter LM: Splinting vs surgery in the treatment of carpal tunnel syndrome: a randomized controlled trial. JAMA 288:1245-1251, 2002

9. Goodrum JF, Earnhardt T, Goines N, Bouldin TW: Fate of myelin lipids during degeneration and regeneration of peripheral nerve: an autoradiographic study. J Neurosci 14:357-367, 1994

10. Gray M, Palispis W, Popovich PG, van Rooijen N, Gupta R: Macrophage depletion alters the blood-nerve barrier without affecting Schwann cell function after neural injury. J Neurosci Res 85:766-777, 2007

11. Gupta R, Channual JC: Spatiotemporal pattern of macrophage recruitment after chronic nerve compression injury. J Neurotrauma 23:216-226, 2006

12. Gupta R, Rowshan K, Chao T, Mozaffar T, Steward O: Chronic nerve compression induces local demyelination and remyelination in a rat model of carpal tunnel syndrome. Exp Neurol 187:500-508, 2004

13. Gupta R, Rummler LS, Palispis W, Truong L, Chao T, Rowshan K, et al: Local down-regulation of myelin-associated glycoprotein permits axonal sprouting with chronic nerve compression injury. Exp Neurol 200:418-429, 2006

14. Gupta R, Steward O: Chronic nerve compression induces concurrent apoptosis and proliferation of Schwann cells. J Comp Neurol 461:174-186, 2003

15. Gupta R, Truong L, Bear D, Chafik D, Modafferi E, Hung CT: Shear stress alters the expression of myelin-associated glycoprotein (MAG) and myelin basic protein (MBP) in Schwann cells. J Orthop Res 23:1232-1239, 2005

16. Jacobson RD, Virag I, Skene JH: A protein associated with axon growth, GAP-43, is widely distributed and developmentally regulated in rat CNS. J Neurosci 6:1843-1855, 1986

17. Johnson PW, Abramow-Newerly W, Seilheimer B, Sadoul R, Tropak MB, Arquint M, et al: Recombinant myelin-associated glycoprotein confers neural adhesion and neurite outgrowth function. Neuron 3:377-385, 1989

18. Le N, Nagarajan R, Wang JY, Araki T, Schmidt RE, Milbrandt 
$\mathrm{J}$ : Analysis of congenital hypomyelinating Egr2Lo/Lo nerves identifies Sox 2 as an inhibitor of Schwann cell differentiation and myelination. Proc Natl Acad Sci U S A 102:2596-2601, 2005

19. Ludwin SK, Maitland M: Long-term remyelination fails to reconstitute normal thickness of central myelin sheaths. J Neurol Sci 64:193-198, 1984

20. Macdonald AG, Fraser PJ: The transduction of very small hydrostatic pressures. Comp Biochem Physiol A Mol Integr Physiol 122:13-36, 1999

21. Mackinnon SE, Dellon AL, Hudson AR, Hunter DA: Chronic human nerve compression-a histological assessment. Neuropathol Appl Neurobiol 12:547-565, 1986

22. McKerracher L, David S, Jackson DL, Kottis V, Dunn RJ, Braun PE: Identification of myelin-associated glycoprotein as a major myelin-derived inhibitor of neurite growth. Neuron 13:805-811, 1994

23. Molliver DC, Wright DE, Leitner ML, Parsadanian AS, Doster $\mathrm{K}$, Wen D, et al: IB4-binding DRG neurons switch from NGF to GDNF dependence in early postnatal life. Neuron 19:849861, 1997

24. Mozaffar T, Strandberg E, Abe K, Hilgenberg LG, Smith MA, Gupta R: Neuromuscular junction integrity after chronic nerve compression injury. J Orthop Res [epub ahead of print], 2008

25. Mukhopadhyay G, Doherty P, Walsh FS, Crocker PR, Filbin MT: A novel role for myelin-associated glycoprotein as an inhibitor of axonal regeneration. Neuron 13:757-767, 1994

26. Neary D, Ochoa J, Gilliatt RW: Sub-clinical entrapment neuropathy in man. J Neurol Sci 24:283-298, 1975

27. Nodari A, Previtali SC, Dati G, Occhi S, Court FA, Colombelli C, et al: Alpha6beta4 integrin and dystroglycan cooperate to stabilize the myelin sheath. J Neurosci 28:6714-6719, 2008

28. O'Brien JP, Mackinnon SE, MacLean AR, Hudson AR, Dellon AL, Hunter DA: A model of chronic nerve compression in the rat. Ann Plast Surg 19:430-435, 1987

29. Parkinson DB, Bhaskaran A, Arthur-Farraj P, Noon LA, Woodhoo A, Lloyd AC, et al: c-Jun is a negative regulator of myelination. J Cell Biol 181:625-637, 2008

30. Parkkinen JJ, Ikonen J, Lammi MJ, Laakkonen J, Tammi M, Helminen HJ: Effects of cyclic hydrostatic pressure on proteoglycan synthesis in cultured chondrocytes and articular cartilage explants. Arch Biochem Biophys 300:458-465, 1993

31. Pestronk A, Drachman DB: Motor nerve terminal outgrowth and acetylcholine receptors: inhibition of terminal outgrowth by alpha-bungarotoxin and anti-acetylcholine receptor antibody. J Neurosci 5:751-758, 1985

32. Poliak S, Peles E: The local differentiation of myelinated axons at nodes of Ranvier. Nat Rev Neurosci 4:968-980, 2003

33. Previtali SC, Feltri ML, Archelos JJ, Quattrini A, Wrabetz L, Hartung H: Role of integrins in the peripheral nervous system. Prog Neurobiol 64:35-49, 2001

34. Rosen HR, Ammer K, Mohr W, Bock P, Kornek GV, Firbas W: Chemically-induced chronic nerve compression in rabbits-a new experimental model for the carpal tunnel syndrome. Langenbecks Arch Chir 377:216-221, 1992

35. Rummler L, Palispis W, Gupta R: A novel hydrostatic compression chamber model for the study of chronic nerve compression injuries in vitro. Presentation to the Society for Neuroscience, November 17, 2008, Washington D.C. (Abstract)

36. Salzer JL: Switching myelination on and off. J Cell Biol 181:575-577, 2008

37. Salzer JL, Bunge RP: Studies of Schwann cell proliferation. I. An analysis in tissue culture of proliferation during development, Wallerian degeneration, and direct injury. J Cell Biol 84:739-752, 1980

38. Schwartz MA, Desimone DW: Cell adhesion receptors in mechanotransduction. Curr Opin Cell Biol, 2008

39. Sommerich CM, Lavender SA, Buford JA: J JB, Korkmaz SV, Pease WS: Towards development of a nonhuman primate model of carpal tunnel syndrome: performance of a voluntary, repetitive pinching task induces median mononeuropathy in Macaca fascicularis. J Orthop Res 25:713-724, 2007

40. Stoll G, Griffin JW, Li CY, Trapp BD: Wallerian degeneration in the peripheral nervous system: participation of both Schwann cells and macrophages in myelin degradation. J Neurocytol 18:671-683, 1989

41. Taskinen HS, Röyttä M: The dynamics of macrophage recruitment after nerve transection. Acta Neuropathol 93:252-259, 1997

42. Tomita K, Kubo T, Matsuda K, Yano K, Tohyama M, Hosokawa $\mathrm{K}$ : Myelin-associated glycoprotein reduces axonal branching and enhances functional recovery after sciatic nerve transection in rats. Glia 55:1498-1507, 2007

43. Topilko P, Schneider-Maunoury S, Levi G, Baron-Van Evercooren A, Chennoufi AB, Seitanidou T, et al: Krox-20 controls myelination in the peripheral nervous system. Nature 371:796-799, 1994

44. Tricaud N, Perrin-Tricaud C, Bruses JL, Rutishauser U: Adherens junctions in myelinating Schwann cells stabilize Schmidt-Lanterman incisures via recruitment of p120 catenin to E-cadherin. J Neurosci 25:3259-3269, 2005

45. Yamaguchi T, Osamura N, Zhao C, Zobitz ME, An KN, Amadio PC: The mechanical properties of the rabbit carpal tunnel subsynovial connective tissue. J Biomech 41:3519-3522, 2007

46. Yoshii Y, Zhao C, Henderson J, Zhao KD, Zobitz ME, An KN, et al: Effects of carpal tunnel release on the relative motion of tendon, nerve, and subsynovial connective tissue in a human cadaver model. Clin Biomech (Bristol, Avon) 23:1121-1127, 2008

Manuscript submitted October 15, 2008.

Accepted November 19, 2008.

Address correspondence to: Ranjan Gupta, M.D., 2226 Gillespie Neuroscience Research Facility, University of California, Irvine, California 92697.email: ranjang@uci.edu. 